\title{
Catalytic Removal of Alizarin Red Using Chromium Manganese Oxide Nanorods: Degradation and Kinetic Studies
}

\author{
Muhammad Hamza ${ }^{1,2}$, Ataf Ali Altaf ${ }^{1,2, *}$, Samia Kausar ${ }^{1,2}$, Shahzad Murtaza ${ }^{1}$, \\ Nasir Rasool ${ }^{3}$, Rukhsana Gul ${ }^{4}$, Amin Badshah ${ }^{5}$, Muhammad Zaheer ${ }^{6}{ }^{D}$, \\ Syed Adnan Ali Shah 7,8 (D) and Zainul Amiruddin Zakaria 9,10,*(D) \\ 1 Department of Chemistry, University of Gujrat, Hafiz Hayat Campus, Gujrat 50700, Pakistan; \\ m.hamzasethi87@gmail.com (M.H.); samiakosar93@gmail.com (S.K.); shahzad.murtaza@uog.edu.pk (S.M.) \\ 2 Department of Chemistry, University of Okara, Okara 56300, Pakistan \\ 3 Department of Chemistry, Government College University, Faisalabad 38000, Pakistan; \\ nasirrasool@gcuf.edu.pk \\ 4 Department of Chemistry, Kohat University of Science and Technology, Kohat 26000, Pakistan; \\ rukh_sanna@yahoo.com \\ 5 Department of Chemistry, Quaid-i-Azam University, Islamabad 45320, Pakistan; aminbadshah@qau.edu.pk \\ 6 Department of Chemistry and Chemical Engineering, SBA School of Science and Engineering, \\ Lahore University of Management Sciences (LUMS), Lahore 54792, Pakistan; \\ muhammad.zaheer@lums.edu.pk \\ 7 Faculty of Pharmacy, Universiti Teknologi MARA Cawangan Selangor Kampus Puncak Alam, \\ Bandar Puncak Alam 42300, Malaysia; syedadnan@uitm.edu.my \\ 8 Atta-ur-Rahman Institute for Natural Products Discovery (AuRIns), Universiti Teknologi MARA Cawangan \\ Selangor Kampus Puncak Alam, Bandar Puncak Alam 42300, Malaysia \\ 9 Department of Biomedical Science, Faculty of Medicine and Health Sciences, Universiti Putra Malaysia, \\ Serdang 43400, Malaysia \\ 10 Halal Institute Research Institute, Universiti Putra Malaysia, Serdang 43400, Malaysia \\ * Correspondence: atafali_altaf@yahoo.com (A.A.A.); zaz@upm.edu.my (Z.A.Z.); \\ Tel.: +92-332-5049532 (A.A.A.); +603-8947-2111 (Z.A.Z.); Fax: +603-8943-6178 (Z.A.Z.)
}

Received: 7 July 2020; Accepted: 28 August 2020; Published: 6 October 2020

\begin{abstract}
Dye removal through photocatalytic degradation employing nanomaterials as catalysts is a growing research area. In current studies, photocatalytic alizarin red (AR) dye degradation has been investigated by designing a series of $\mathrm{Cr}$ based manganese oxide nanomaterials (MH1-MH5). Synthesized nanomaterials were characterized by powder X-ray diffraction, scanning electron microscopy/energy dispersive $\mathrm{x}$-ray, Brunauer-Emmett-Teller, and photoluminescence techniques and were utilized for photocatalytic AR dye degradation under UV light. AR dye degradation was monitored by UV-visible spectroscopy and percent degradation was studied for the effect of time, catalyst dose, different dye concentrations, and different $\mathrm{pH}$ values of dye solution. All the catalysts have shown more than $80 \%$ dye degradation exhibiting good catalytic efficiencies for dye removal. The catalytic pathway was analyzed by applying the kinetic model. A pseudo second-order model was found the best fitted kinetic model indicating a chemically-rate controlled mechanism. Values of constant $\mathrm{R}^{2}$ for all the factors studied were close to unity depicting a good correlation between experimental data.
\end{abstract}

Keywords: alizarin red (AR); photocatalysis; degradation; UV Light; nanomaterials; kinetics 


\section{Introduction}

Textile dyes produce large amounts of effluents having contaminants that are often discharged to surface water bodies and groundwater aquifers [1]. These discharges are a major source of concern as they have considerable potential to disturb the local and downstream ecosystems. Printing, textile, papermaking, pharmaceutical, food processing, and cosmetics industries can all be sources of effluent containing significant concentrations of dyes and pigments [2-5]. Most of the dyes which are being used in the textile industries are usually stable and nonbiodegradable [6]. These dyes belong to the class of synthetic organic compounds and are considered as major environmental pollutants due to their large-scale production, high aromaticity, chemical stability and toxicity [7-9]. To reduce the risk of environmental pollution, it is necessary to treat wastewater reserves before discharging them into the environment [10]. Various physical and chemical processes have been employed for dye degradation in wastewater treatment. However, these processes cannot be effectively employed to treat the wide range of wastewater effluents for dye degradation due to their high operation costs, energy consumption, reduced efficiency due to the chemical stability of dyes and/or the complexity of their degradation [11].

However, the recent trend is to combine techniques for sustainability, as an oxidation/catalysis or adsorption/catalysis to overcome the drawbacks of conventional techniques [12]. The photocatalytic degradation of dyes by nanoparticles as catalysts is a growing research area due to its quick oxidation, no formation of polycyclic products, and oxidation of pollutants [13]. Particles in the nanometer size range have a very large surface area, thereby offering many binding sites to the reacting species. A huge number of transition metal oxides catalysts were synthesized and employed for dye degradation purposes employing direct sunlight or UV light [14]. Metal oxide-based nanomaterials are widely employed due to their flexibility to modulate several characteristics-i.e., structure, composition, morphology, and surface area enhancements [15-17]. Oxides like $\mathrm{TiO}_{2}, \mathrm{ZnO}$, and CdS are effective with UV light, easily available, relatively inexpensive and chemically stable photocatalysts [18-21]. Doped/undoped manganese oxides nanomaterials are promising materials due to their low cost, high stability, and good environmental compatibility [22].

Alizarin red (AR) has been widely employed in the textile industry [23]. It is a major textile effluent and has been used for the photocatalytic degradation of alizarin red in an aqueous medium [24]. Various studies were performed for photodegradation of AR dye using various photocatalysts. Joshi and Shrivastava used $\mathrm{ZnO}$ and $\mathrm{TiO}_{2}$ as photocatalysts for AR dye degradation under UV and attained maximum removal in 200 min with very low initial dye concentration (20-60 ppm) [25]. Kansal et al. [26] used $\mathrm{ZnO}$ nanoparticles for AR dye degradation and achieved 77\% degradation in 90 min by using a UV chamber.

In the present study, chromium-doped manganese oxide nanomaterials (MH1-MH5) were synthesized by the hydrothermal method. The effect of the time of contact between the synthesized nanomaterials and the AR dye, different catalyst doses, various dye concentrations in the aqueous solutions, and variating $\mathrm{pH}$ of the dye solutions on nanomaterials were investigated. The kinetic parameters have been evaluated to study the reaction mechanisms.

\section{Results and Discussion}

\subsection{Characterization of Nanomaterials (MH1-MH5)}

\subsubsection{Elemental Analysis by EDX}

The elemental analysis of all the synthesized materials (MH1-MH5) was carried out by energy dispersive X-ray (EDX) analysis technique coupled with scanning electron microscopy (SEM). The spectrographs obtained in this case are presented in supplemental data (Figure S1). The elemental composition found in this respect is reported in Table 1 along with other parameters. 
Table 1. Synthetic conditions, powder X-ray diffraction (PXRD) peaks and found composition (energy dispersive X-ray; EDX) of synthesized nanomaterials (MH1-MH5).

\begin{tabular}{|c|c|c|c|c|}
\hline Samples & Found Unit Formula & Synthetic Conditions & 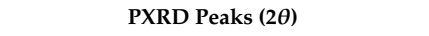 & Found Composition by EDX \\
\hline MH1 & $\mathrm{Mn}_{0.99} \mathrm{Cr}_{0.1} \mathrm{O}_{2}$ & $\begin{array}{c}0.948 \mathrm{~g} \mathrm{KMnO}_{4}, 0.24 \mathrm{~g} \\
\mathrm{~K}_{2} \mathrm{Cr}_{2} \mathrm{O}_{7}, 20 \mathrm{~mL} \mathrm{HCl}\end{array}$ & $\begin{array}{c}12.65,17.89,28.59,37.33,41.77,49.59,56.31, \\
59.87,69.37\end{array}$ & $\mathrm{Mn}$ (54.69); $\mathrm{Cr}$ (6.18); O (39.09) \\
\hline MH2 & $\mathrm{Mn}_{0.87} \mathrm{Cr}_{0.15} \mathrm{O}_{2}$ & $\begin{array}{l}0.948 \mathrm{~g} \mathrm{KMnO}_{4}, 0.41 \mathrm{~g} \\
\mathrm{~K}_{2} \mathrm{Cr}_{2} \mathrm{O}_{7}, 20 \mathrm{~mL} \mathrm{HCl}\end{array}$ & $\begin{array}{c}12.61,18.03,28.71,37.41,41.79,49.67,59.93 \\
69.31\end{array}$ & $\mathrm{Mn}(48.05) ; \mathrm{Cr}(8.26) ; \mathrm{O}(43.68)$ \\
\hline MH3 & $\mathrm{Mn}_{0.99} \mathrm{Cr}_{0.13} \mathrm{O}_{2}$ & $\begin{array}{l}0.948 \mathrm{~g} \mathrm{KMnO}_{4}, 0.35 \mathrm{~g} \\
\mathrm{~K}_{2} \mathrm{Cr}_{2} \mathrm{O}_{7}, 20 \mathrm{~mL} \mathrm{HCl}\end{array}$ & $\begin{array}{c}12.67,18.09,28.77,37.41,41.85,49.71,56.29 \\
60.11,69.33\end{array}$ & $\mathrm{Mn}$ (54.88); $\mathrm{Cr}$ (7.21); O (37.90) \\
\hline MH4 & $\mathrm{Mn}_{0.96} \mathrm{Cr}_{0.09} \mathrm{O}_{2}$ & $\begin{array}{l}0.948 \mathrm{~g} \mathrm{KMnO}_{4}, 0.18 \mathrm{~g} \\
\mathrm{~K}_{2} \mathrm{Cr}_{2} \mathrm{O}_{7}, 20 \mathrm{~mL} \mathrm{HCl}\end{array}$ & $\begin{array}{c}13.09,18.39,28.85,37.73,42.07,50.13,56.67 \\
60.25,70.03\end{array}$ & $\mathrm{Mn}(53.05) ; \mathrm{Cr}(5.05) ; \mathrm{O}(41.88)$ \\
\hline MH5 & $\mathrm{Mn}_{0.92} \mathrm{Cr}_{0.12} \mathrm{O}_{2}$ & $\begin{array}{l}0.948 \mathrm{~g} \mathrm{KMnO}_{4}, 0.30 \mathrm{~g} \\
\mathrm{~K}_{2} \mathrm{Cr}_{2} \mathrm{O}_{7}, 20 \mathrm{~mL} \mathrm{HCl}\end{array}$ & $\begin{array}{c}12.77,18.05,28.65,37.45,41.95,49.79,56.25 \\
60.13,69.03\end{array}$ & $\mathrm{Mn}$ (50.69); $\mathrm{Cr}$ (6.41); O (42.88) \\
\hline
\end{tabular}

The results in Table 1 for nanomaterials (MH1-MH5) indicate that the analyzed elemental compositions are in close agreement with the expected elemental percentages in accordance with the reactants used for synthesis. The high-intensity peaks of Mn for MH1-MH5 indicate the presence of $\mathrm{Mn}$ with a major elemental composition, with percentage ranging from 48.05 to $54.88 \%$. For the percentage composition ranges for $\mathrm{Cr}(5.06-6.26 \%)$ and $\mathrm{O}(37.90-43.68 \%)$, a minor presence was observed. EDX patterns are shown for MH1-MH5 in supplemental data (Figure S1).

\subsubsection{Powder X-Ray Diffraction (PXRD) Analysis}

The phase composition and particle size of the synthesized materials were characterized by PXRD and the data obtained are reported in Table 2. The PXRD patterns of nanomaterials (MH1-MH5) are presented in Figure 1. Powder patterns for samples were matched to standard patterns of nanomaterials. Specific hkl values were evaluated corresponding to 2 theta values. These hkl values were matched with standard PXRD patterns for $\alpha-\mathrm{MnO}_{2}$ (JCPD No.44-0141), (JCPD No. 01-1127), $\mathrm{CrO}_{3}$ (JCPD No. 74-0326, 70-3766). The PXRD of (MH1-MH5) depicted the presence of $\alpha-\mathrm{MnO}_{2}$ as a major phase with slight incorporation of $\mathrm{CrO}_{3}$ and $\mathrm{MnCrO}_{4}$. It was indicated by the powder patterns that the peak for $\alpha-\mathrm{MnO}_{2}$ showed a gradual decrease in intensity as the concentration of $\mathrm{Cr}$ increased from $\mathrm{MH} 1$ to MH5 indicated by the peaks for $\mathrm{CrO}_{3}$. The major phase $\alpha-\mathrm{MnO}_{2}$ is tetragonal in structure having P4_2/mnm space group. These peak assignments in PXRD jointly with EDX results indicate that the MH1-MH5 samples are monocrystalline materials with the elemental composition given in Table 2.

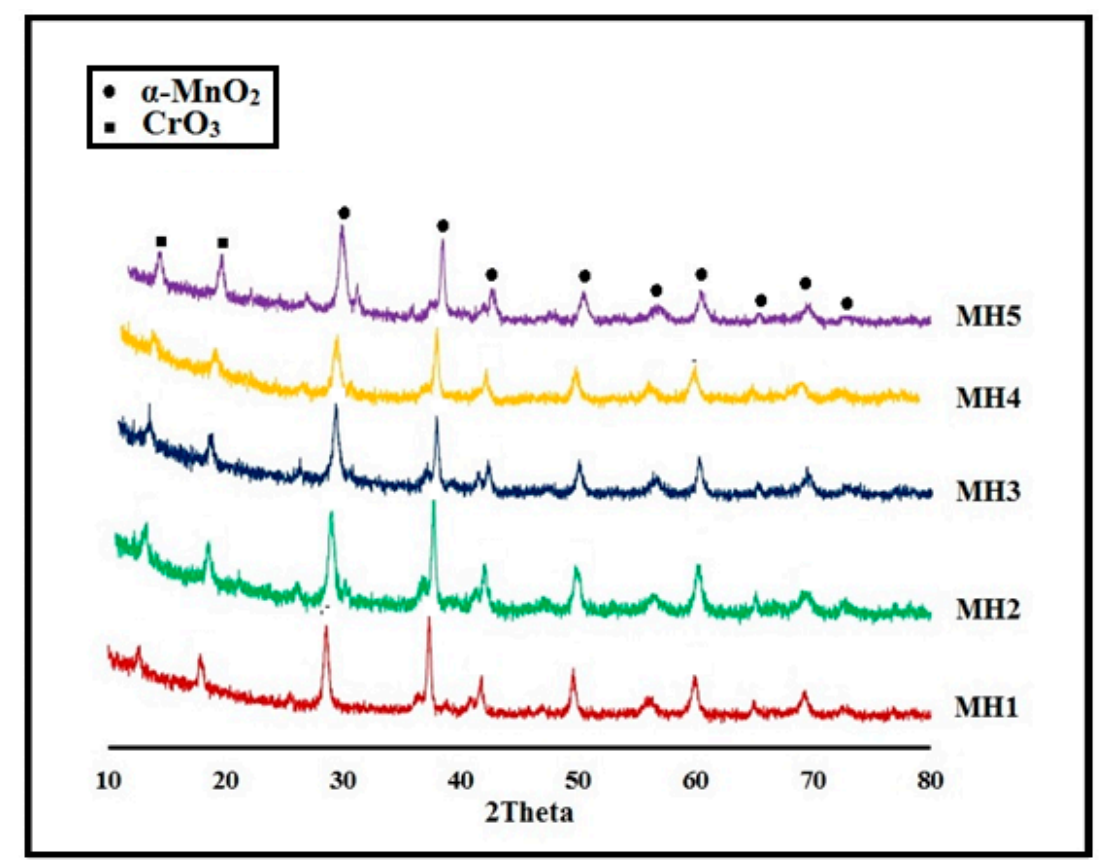

Figure 1. PXRD pattern of nanomaterials (MH1-MH5). (Powder patterns are matched with standard patterns-i.e., $\alpha-\mathrm{MnO}_{2}$ ((JCPD No.44-0141), (JCPD No. 01-1127), $\mathrm{CrO}_{3}$ (JCPD No. 74-0326, 70-3766)). 
Table 2. Synthetic conditions, composition and PXRD parameters of nanomaterials (MH1-MH6).

\begin{tabular}{|c|c|c|c|c|c|}
\hline Samples & Found Composition & $\begin{array}{c}\text { Average Crystallite Size } \\
\text { D (nm) }\end{array}$ & Volume $\mathrm{V}=\mathrm{D}^{3}$ & Dislocation Density $\times 10^{-3}(\mathrm{~nm})^{-2}(\delta)$ & $\begin{array}{l}\text { Micro Strain } \\
(\varepsilon)\end{array}$ \\
\hline MH1 & $\mathrm{Mn}_{0.99} \mathrm{Cr}_{0.1} \mathrm{O}_{2}$ & $44.02 \pm 8$ & 82,780 & $5.95 \times 10^{-4}$ & 0.035 \\
\hline MH2 & $\mathrm{Mn}_{0.87} \mathrm{Cr}_{0.15} \mathrm{O}_{2}$ & $38.90 \pm 6$ & 62,205 & $6.98 \times 10^{-4}$ & 0.038 \\
\hline MH3 & $\mathrm{Mn}_{0.99} \mathrm{Cr}_{0.13} \mathrm{O}_{2}$ & $49.60 \pm 5$ & 126,625 & $4.19 \times 10^{-4}$ & 0.027 \\
\hline MH4 & $\mathrm{Mn}_{0.96} \mathrm{Cr}_{0.09} \mathrm{O}_{2}$ & $50.22 \pm 7$ & 135,584 & $4.20 \times 10^{-4}$ & 0.02 \\
\hline MH5 & $\mathrm{Mn}_{0.92} \mathrm{Cr}_{0.12} \mathrm{O}_{2}$ & $36.82 \pm 3$ & 49,576 & $7.60 \times 10^{-4}$ & 0.035 \\
\hline
\end{tabular}

After the PXRD phase assignments, other parameters-i.e., the crystallite size, dislocation densities, crystallite volume, and microstrain, were calculated for all the synthesized materials and are reported in Table 2. The particle size calculation was performed using the Debye Sherrer formula [27] as follows:

$$
\mathrm{D}=0.91 \lambda / \beta \cos \theta
$$

where $\mathrm{D}$ is the particle size of the sample and $\lambda$ is the wavelength of the $\mathrm{X}$-ray diffraction beam and has a value of 1.5405980 . The angle at which diffraction occurs is $\cos \theta$, and $\beta$ is the FWHM (full width at half maximum) value [28].

The volume $(\mathrm{V})$ of crystalline material was calculated using the relation $\left(V=D^{3}\right)$ [29]. The dislocation density $(\delta)$, which represents the extent of defects in the sample, is defined as the length of the dislocation lines per unit volume of the crystal and is calculated using Equation (2) [30].

$$
\delta=1 / \mathrm{D}^{2}
$$

The strain-induced broadening in powders due to crystal imperfection and distortion is calculated as a microstrain $(\varepsilon)$ [31]. Positive values of $\varepsilon$ represent a tensile strain while negative values represent a compressive strain. Strain-induced broadening (microstrain) in nanomaterials was calculated by Equation (3) [31] as follows:

$$
\varepsilon=\beta / 4 \tan \theta
$$

The average crystallite sizes for (MH1-MH5) at their corresponding intensities were 44.02 for MH1, 38.90 for MH2, 49.60 for MH3, 50.22 for MH4 and 36.82 for MH5. It was noted that the crystallite size is increased by the inclusion of chromium which causes the crystal stress as depicted in Table 2. The values of dislocation densities show the extent of defects, which are fewer in nanomaterials (MH1-MH5). Nanomaterials with smaller crystallite sizes showing greater stabilities. Values for crystalline volume indicates that there is proper incorporation of chromium ions into regular lattice sites of $\mathrm{O}^{-}$in the samples. The inclusion of $\mathrm{Cr}$ ions in the $\alpha-\mathrm{MnO}_{2}$ structure causes an increase in the strain and increases crystal imperfections. Positive values depicted the tensile microstrain in MH1-MH5 nanomaterials which can be due to the lattice shrinkage.

\subsubsection{Scanning Electron Microscopy (SEM) Analysis}

Surface morphological characteristics were analyzed for all the synthesized series of nanomaterials by SEM and are reported in Table 3. SEM images of representative material MH5 are shown in Figure 2 while other nanomaterials (MH1-MH4) are enlisted in the supplemental data (Figure S2a-d) and the surface morphological characteristics are reported in Table 3. SEM images indicated that synthesized nanomaterials (MH1-MH5) are crystalline and monodisperse. The surface morphological characteristics indicated crystalline rod-like structures for all the synthesized nanomaterials. 
Table 3. Morphological characteristics by SEM analysis of nanomaterials (MH1-MH5).

\begin{tabular}{cllc}
\hline Samples & Material Nature & Dispersity & Structural Appearance \\
\hline MH1 & Monocrystalline & Monodisperse & Elongated Rod-like Structures \\
MH2 & Monocrystalline & Monodisperse & Elongated Rod-like Structures \\
MH3 & Monocrystalline & Monodisperse & Elongated Rod-like Structures \\
MH4 & Monocrystalline & Monodisperse & Short Edged Rods \\
MH5 & Monocrystalline & Monodisperse & Elongated Rod-like Structures \\
\hline
\end{tabular}
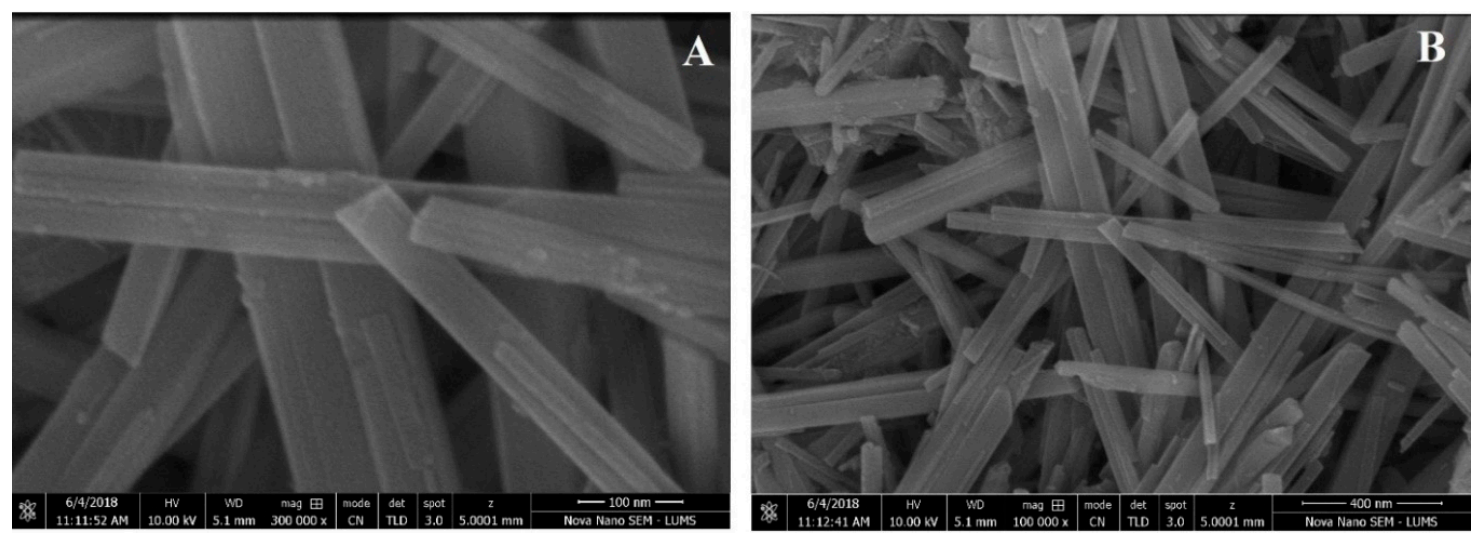

Figure 2. SEM images of the representative sample MH5 ((A) $(100 \mathrm{~nm})$ and (B) $(400 \mathrm{~nm}))$.

\subsubsection{Brunauer-Emmett-Teller (BET) Analysis}

The specific surface area of nanomaterials (MH1-MH5), calculated by multipoint BET, and pore structure parameters were calculated from the DFT method (based on molecular modelling and takes into account direct interaction of adsorbate with the adsorbent surface) and are enlisted in Table 4. The specific surface area of nanomaterials was in the range of $20-46 \mathrm{~m}^{2} / \mathrm{g}$. MH3 has the maximum surface area of $46.32 \mathrm{~m}^{2} / \mathrm{g}$, whereas MH5 has a surface area of $20.45 \mathrm{~m}^{2} / \mathrm{g}$ but smaller pore volumes- 0.038 and 0.032 , respectively -indicating materials were microporous. Values of the constant (C) for $\mathrm{MH} 2, \mathrm{MH} 3$, and $\mathrm{MH} 4$ were $<100$, which indicates a strong adsorbate-adsorbent interaction. For MH1 and MH5, the values are high due to the high porosity of the material. Comparison of the experimental data with the BET results showed that varying the $\mathrm{Cr}$ concentration during the synthesis of nanomaterials (Section 2.2), significantly affected the specific surface area and pore size distribution of nanomaterials.

Table 4. Brunauer-Emmett-Teller (BET) properties of synthesized nanomaterials (MH1-MH5).

\begin{tabular}{cccccc}
\hline \multirow{2}{*}{ BET Properties } & MH1 & MH2 & MH3 & MH4 & MH 5 \\
\cline { 2 - 6 } & 37.153 & 33.998 & 46.325 & 37.515 & 20.906 \\
\hline Surface ARea $\left(\mathbf{S}_{\mathbf{B E T}}\right)\left(\mathbf{m}^{\mathbf{2}} / \mathbf{g}\right)$ & 0.048 & 0.018 & 0.038 & 0.043 & 0.032 \\
Pore Volume $\left(\mathbf{V}_{\mathbf{m}}\right)(\mathbf{c c} / \mathbf{g})$ & 3.775 & 2.425 & 2.583 & 3.537 & 4.887 \\
Pore Width $(\mathbf{n m})$ & 6951.982 & 4.652 & 19.11 & 93.29 & 2090.61 \\
Constant C & & & & & \\
\hline
\end{tabular}

\subsubsection{Photoluminescence (PL) Study}

The PL emission spectra of nanomaterials (MH1-MH5) have been obtained through a PL spectrophotometer with an exciton wavelength of $420 \mathrm{~nm}$. The Cr-doped manganese oxide nanomaterials exhibited some sharp and broad PL peaks in the visible region from 420 to $700 \mathrm{~nm}$. Figure 3 shows the PL spectra of nanomaterials (MH1-MH5) giving prominent peaks at 435, 452, 488,588 and $630 \mathrm{~nm}$ corresponding to $2.85,2.74,2.54,2.11$ and $1.97 \mathrm{eV}$ energy range. In this region, the emission results from the radiative recombination of the photogenerated hole with an electron 
occupying oxygen vacancy [32]. An increase in the emitted intensity in the visible region was observed in PL peaks with increasing Cr-doping [33] which most probably originated from the near band edge (NBE) due to the recombination of free exciton through exciton-exciton collision processes [32]. The narrower PL peaks also designate a lower trap density [34] in the synthesized materials which is advantageous for them to be applied as efficient photocatalysts [35]. Hence, synthesized nanomaterials may show prominent catalytic efficiencies.

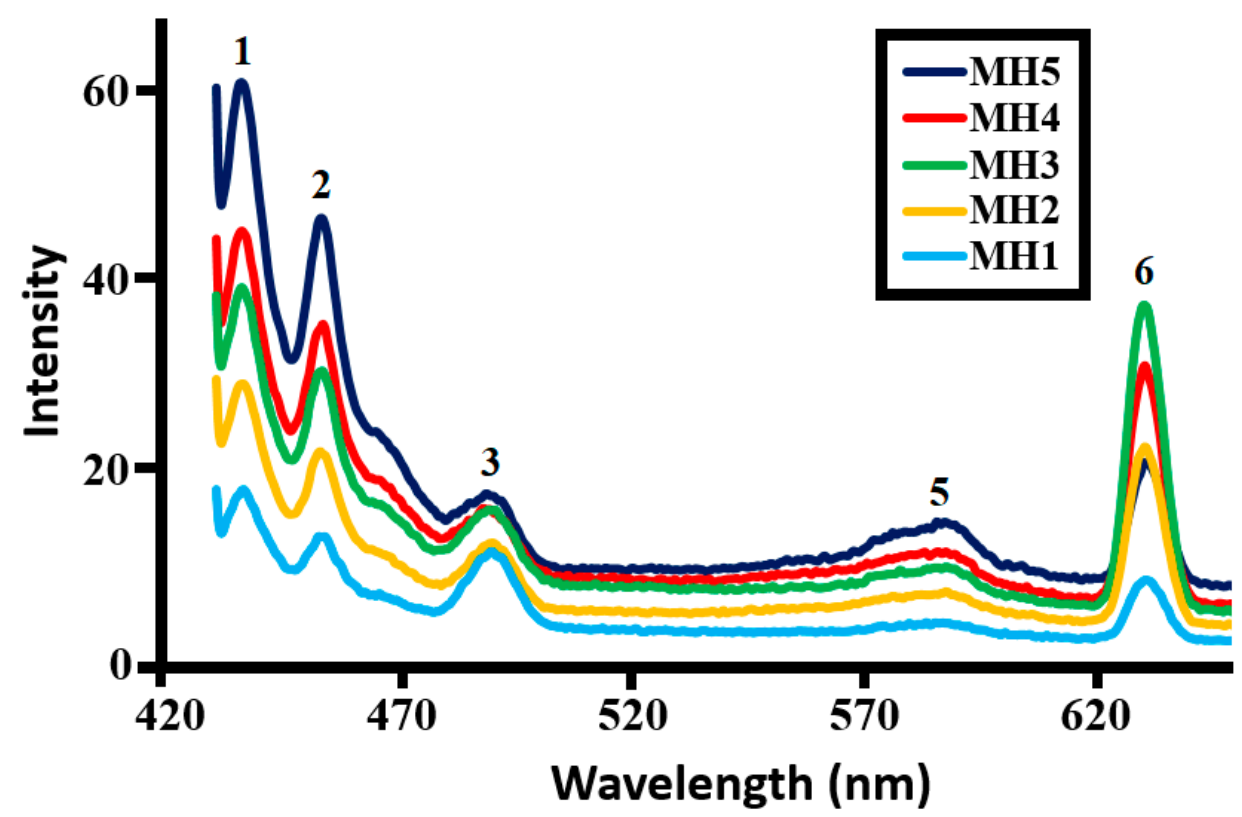

Figure 3. Photoluminescence (PL) spectra of the nanomaterials (MH-MH5).

\subsection{Catalytic Activity Studies}

\subsubsection{Photocatalytic Degradation of AR by Nanocatalysts (MH1-MH5)}

Photocatalytic AR dye degradation activity (see Section 3.4) carried out under a UV light and sample aliquots were taken at different time intervals. Absorbance at $505 \mathrm{~nm}$ was measured by the UV spectrophotometer for all the aliquots taken during the photocatalytic AR dye degradation under the UV light at different time intervals. For the absorbance, the percentage of dye degradation was calculated as described in Section 3.4. The peaks at $505 \mathrm{~nm}$ tend to decrease with the increase in the photocatalytic reaction time indicating the decomposition of the aromatic ring. Similarly, different factors like the effect of time, catalytic dose, initial dye concentrations, and $\mathrm{pH}$ were investigated by calculating the percentage of dye degradation.

The absorption spectrum of the dye solution (without catalyst) before and after keeping it without UV light irradiation for $180 \mathrm{~min}$ is given in Figure S3a (supplemental data) whereas the UV spectra of AR solution at start and after 180 min of UV light irradiation is given in Figure S3b (supplemental data). No change was observed in the AR dye solution after keeping it 180 min without UV light irradiation whereas a very minute change was observed for the dye solution irradiated with UV light for $180 \mathrm{~min}$. The photocatalytic AR dye degradation with all synthesized nanomaterials (MH1-MH5) at various time intervals was calculated and showed more than $80 \%$ degradation of dye as shown in Figure S4 (supplemental data). Hence, the effect of different factors on the percent dye degradation is elaborated for most active catalysts in discussion below.

\subsubsection{Effect of Time}

Photocatalytic dye degradation under UV light was investigated at different time intervals-i.e., $30,60,90,120,150$, and $180 \mathrm{~min}$ and the percentage of dye degradation values calculated are shown in 
Table 5. Nanomaterials (MH1-MH5) have shown a degradation of more than $40 \%$ dye after $30 \mathrm{~min}$ of photocatalytic dye degradation under UV light. An increase in the percentage of dye degradation was observed with every increasing time interval. A dye degradation of more than $80 \%$ was calculated at the end of photocatalytic activity by nanomaterials. Among all the nanomaterials MH1 and MH5 showed maximum photocatalytic efficiencies with 88.99 and $88.89 \%$ dye degradation, respectively (Figure 4). The correlation between the percentage of dye degradation and photocatalytic activity time was found to be significant as indicated by $R^{2}$ values (Figure 5 ). The correlation between the percentage of dye degradation and photocatalytic activity time for (MH2-MH4) are presented in Figure S5 (supplemental data).

Table 5. Photocatalytic degradation of alizarin red (AR) dye (250 ppm) by MH1-MH5 (0.025 g) at different time intervals.

\begin{tabular}{cccccc}
\hline Time (min) & MH1 & MH2 & MH3 & MH4 & MH5 \\
\hline $\mathbf{3 0}$ & 46.21 & 44.94 & 45.61 & 44.21 & 47.23 \\
$\mathbf{6 0}$ & 57.11 & 52.40 & 52.12 & 53.02 & 54.15 \\
$\mathbf{9 0}$ & 61.78 & 61.00 & 62.09 & 63.07 & 62.20 \\
$\mathbf{1 2 0}$ & 67.78 & 66.18 & 68.21 & 68.22 & 67.98 \\
$\mathbf{1 5 0}$ & 79.29 & 78.79 & 78.76 & 79.51 & 77.13 \\
$\mathbf{1 8 0}$ & 89.06 & 88.99 & 86.76 & 88.23 & 89.89 \\
\hline
\end{tabular}
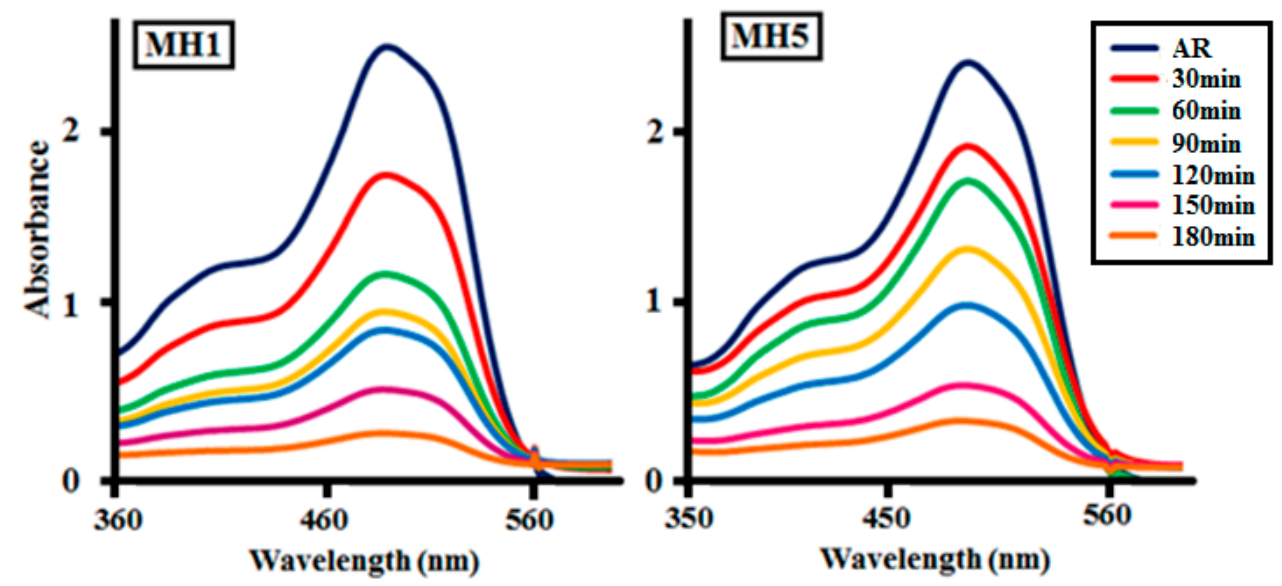

Figure 4. Photocatalytic degradation of alizarin red (AR) dye (250 ppm) at different time intervals by $0.025 \mathrm{~g}$ of MH1 and MH5.

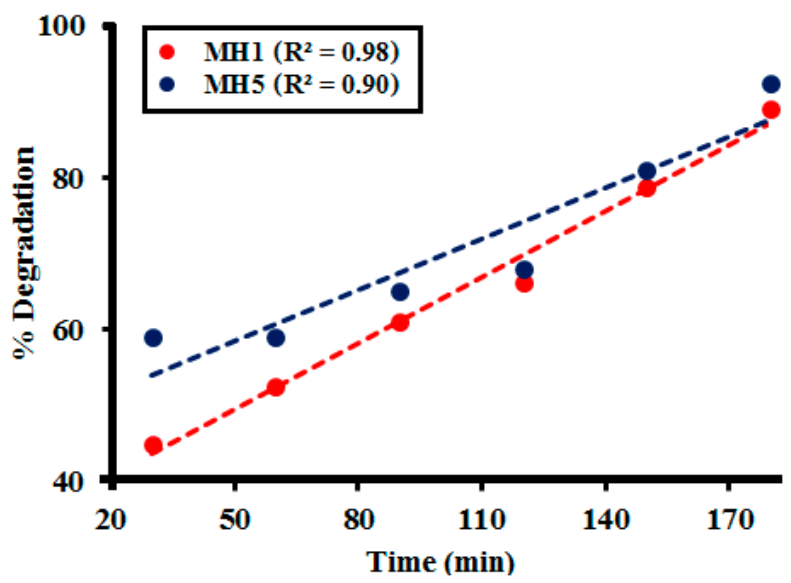

Figure 5. Correlation between time and percentage degradation of alizarin red (AR) dye ( $250 \mathrm{ppm})$ by $0.025 \mathrm{~g}$ of nanomaterials MH1 and MH5. 


\subsubsection{Effect of Photocatalyst Dosage}

The effect of the catalyst dose on the dye degradation was investigated by changing the catalysts (MH1-MH5) dosage-i.e., 0.020, 0.025, 0.030, 0.035, and $0.040 \mathrm{~g} / \mathrm{L}$ for $60 \mathrm{~min}$ irradiation of UV light as shown in Figure 6. The percent degradation of the AR dye (200 ppm) was calculated for (MH1-MH5) nanomaterials and is given in Table 6 . The percentage of dye degradation was found increase with increasing catalyst dose from $0.020 \mathrm{~g} / \mathrm{L}$ to $0.040 \mathrm{~g} / \mathrm{L}$ for nanomaterials. An increase in the percentage of dye degradation was observed by increasing the dose of the catalysts. A dye degradation of more than $38 \%$ was observed for $0.020 \mathrm{~g}$ of catalysts (MH1-MH5) dose which got increased to more than $80 \%$ for all the nanomaterials by increasing dose to $0.040 \mathrm{~g}$. The highest dye degradations $(83.78$ and $84.34 \%$ ) were achieved after $60 \mathrm{~min}$ irradiation of UV light for dosage $0.040 \mathrm{~g} / \mathrm{L}$ of MH4 and MH5. The maximum increase in the dosage of nanomaterials (MH1-MH5) $(0.040 \mathrm{~g} / \mathrm{L})$ results in a maximum dye degradation, possibly contributed by an increased surface provided by catalyst resulting in more photons AR arriving at the surface of catalysts. The correlation between the percentage of dye degradation and catalysts doses were found to be significant as the $R^{2}$ values calculated were close to 1 (Figure 7). The correlations between the percentage of dye degradation and catalyst dose for (MH1-MH3) are presented in Figure S6 (supplementary data).

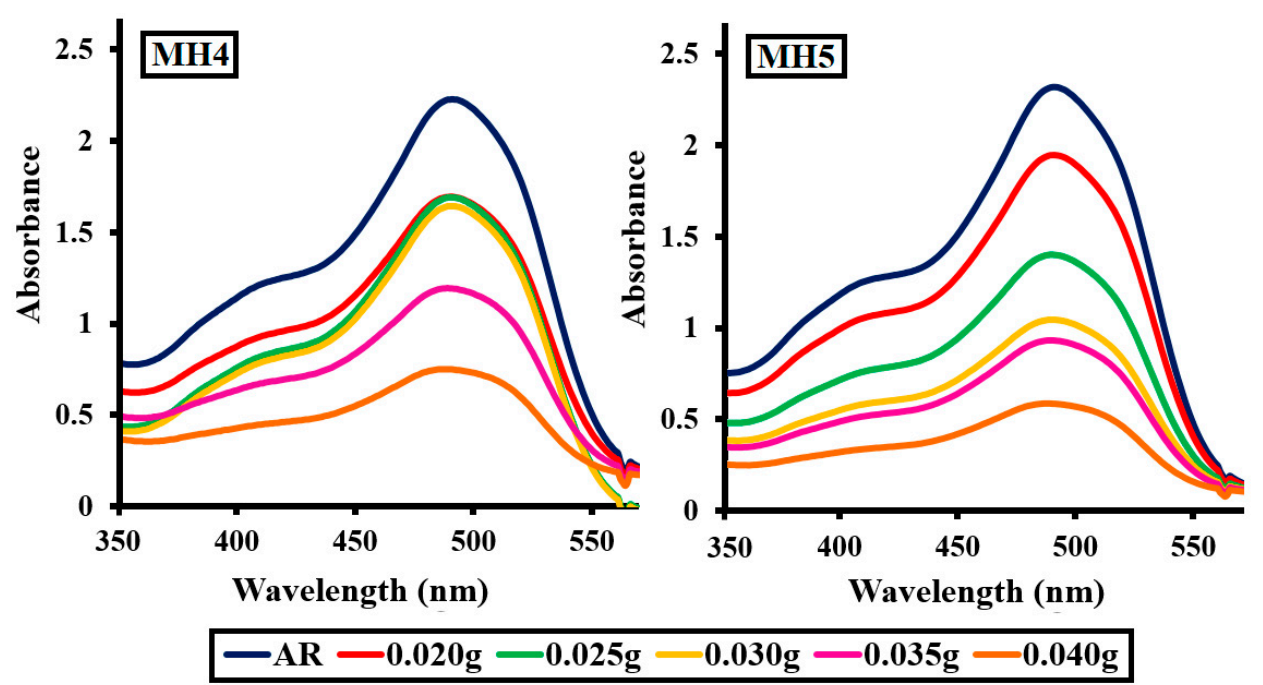

Figure 6. Photocatalytic degradation of alizarin red (AR) dye (250 ppm) at different catalyst doses of MH4 and MH5 after 60 min of photocatalytic activity.

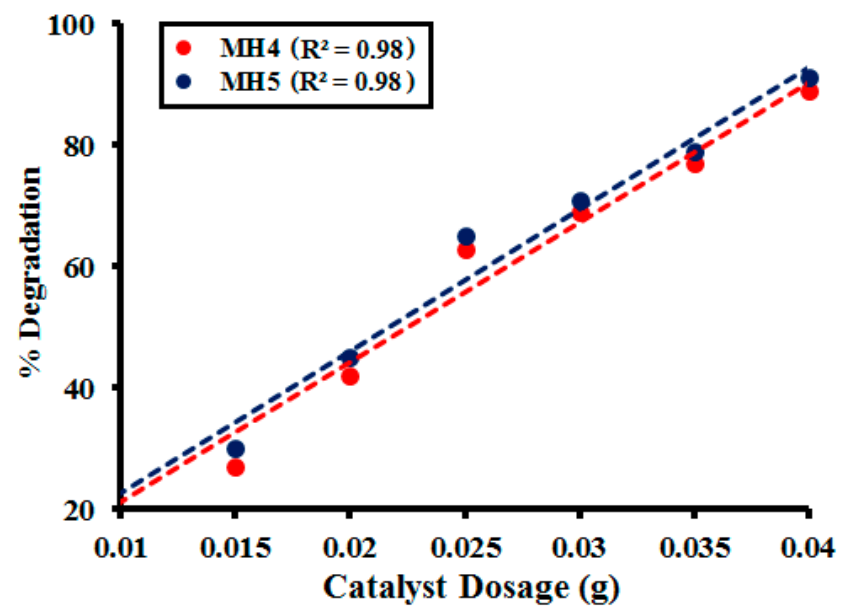

Figure 7. Correlation between different catalyst doses of MH4 and MH5 and percentage degradation of alizarin red (AR) Dye (250 ppm) after $60 \mathrm{~min}$ of photocatalytic activity. 
Table 6. Photocatalytic degradation of alizarin red (AR) dye ( $250 \mathrm{ppm})$ by different catalytic dosage of MH1-MH5 after 60 min of photocatalytic activity.

\begin{tabular}{cccccc}
\hline Amounts (g) & MH1 & MH2 & MH3 & MH4 & MH5 \\
\hline $\mathbf{0 . 0 2 0}$ & 38.99 & 39.23 & 38.32 & 40.22 & 41.77 \\
$\mathbf{0 . 0 2 5}$ & 57.11 & 52.40 & 52.12 & 53.02 & 54.15 \\
$\mathbf{0 . 0 3 0}$ & 67.32 & 68.23 & 66.21 & 67.88 & 68.54 \\
$\mathbf{0 . 0 3 5}$ & 70.86 & 69.54 & 69.86 & 70.55 & 71.87 \\
$\mathbf{0 . 0 4 0}$ & 81.76 & 82.87 & 81.36 & 83.78 & 84.34 \\
\hline
\end{tabular}

\subsubsection{Effect of Dye Concentration}

The effect of AR dye solution concentration on its degradation percentage was investigated by changing the solution concentrations-i.e., 50, 100, 150, 200, and $250 \mathrm{ppm}-$ for photocatalytic activity with 60 min UV light irradiation (shown in Figure 8). The percentage of dye degradation of different AR dye solution concentrations was calculated for (MH1-MH5) nanomaterials and is given in Table 7. The percentage of dye degradation of AR was found to be decreased with increasing concentrations of dye solution from $50 \mathrm{ppm}$ to $250 \mathrm{ppm}$ for nanomaterials. More than $80 \%$ dye degradation was observed for when 50 ppm solution of AR was employed for catalysts (MH1-MH5), which decreased to $60 \%$ for all the nanomaterials with increasing solution concentrations up to $250 \mathrm{ppm}$.

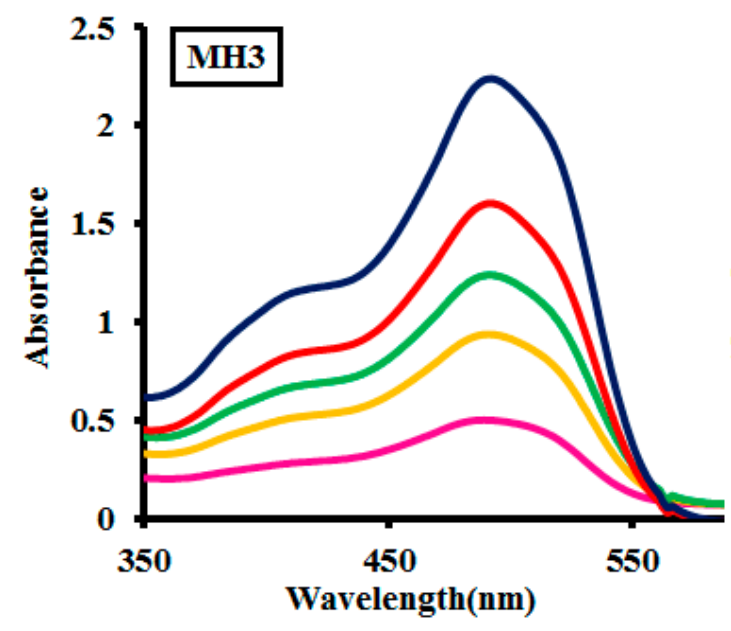

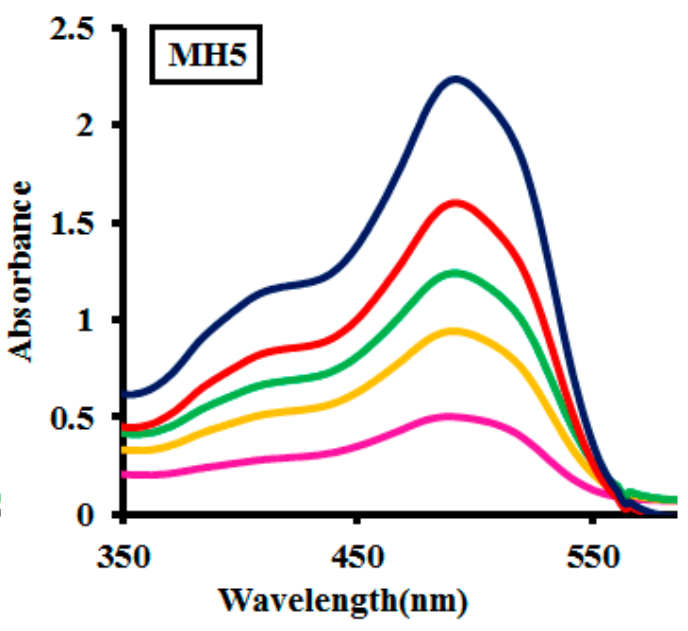

Wavelength(nm)

\section{$-50 p p m-100 p p m-150 p p m-200 p p m-250 p p m$}

Figure 8. Photocatalytic degradation of alizarin red (AR) dye at different dye concentrations (50-250 ppm) by $0.025 \mathrm{~g}$ of MH3 and MH5 after $60 \mathrm{~min}$ of photocatalytic activity.

Table 7. Photocatalytic degradation of different concentrations (50-250 ppm) of alizarin red (AR) dye by MH1-MH5 (0.025 g) after 60 min of photocatalytic activity.

\begin{tabular}{cccccc}
\hline Dye Concentration (ppm) & MH1 & MH2 & MH3 & MH4 & MH5 \\
\hline $\mathbf{5 0}$ & 82.12 & 83.09 & 83.87 & 83.43 & 83.54 \\
$\mathbf{1 0 0}$ & 77.47 & 77 & 79.22 & 78.87 & 78.12 \\
$\mathbf{1 5 0}$ & 70.47 & 71 & 71.12 & 70.45 & 72.22 \\
$\mathbf{2 0 0}$ & 63.21 & 65.03 & 65.83 & 64.22 & 64.08 \\
$\mathbf{2 5 0}$ & 57.11 & 52.40 & 52.12 & 53.02 & 54.95 \\
\hline
\end{tabular}

The highest dye degradations $(83.87 \%$ and $83.54 \%)$ were achieved after 60 min irradiation of UV light for $50 \mathrm{ppm}$ of dye solution by catalysts MH3 and MH5. In contrast, increasing dye concentrations gradually from $50 \mathrm{ppm}$ to $250 \mathrm{ppm}$ tended to decrease the percentage of dye degradation to $61.11 \%$ for $\mathrm{MH} 3$ and $63.21 \%$ for MH5. The correlation between the percentage of dye degradation and 
dye concentrations was found to be significant as the $R^{2}$ value calculated was close to 1 (Figure 9). The correlation between the percentage of dye degradation and different dye concentrations for (MH1-MH3) are presented in Figure S7 (supplemental data).

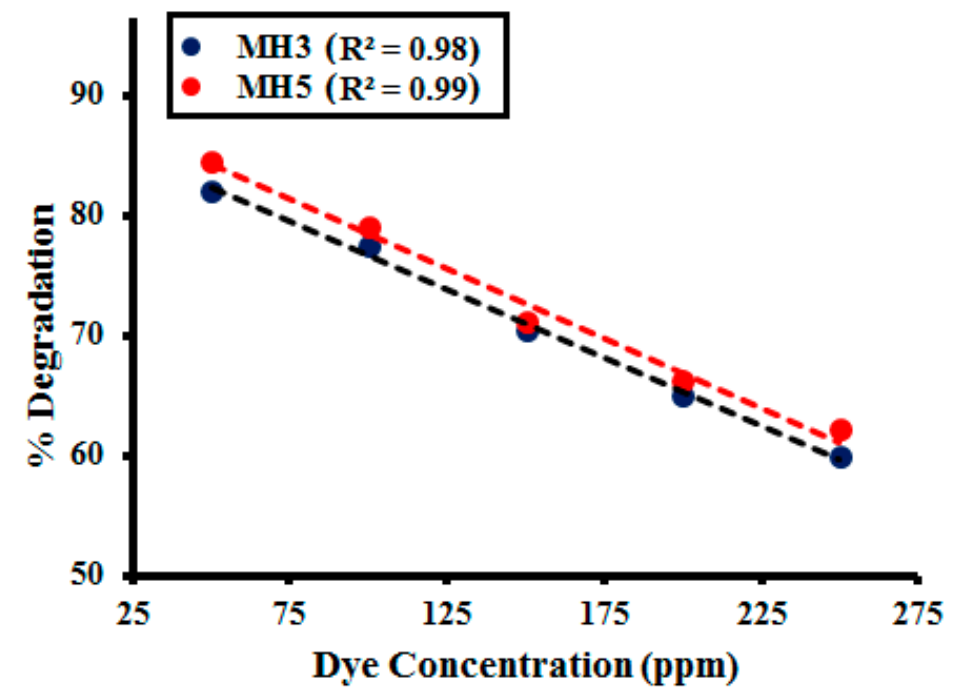

Figure 9. Correlation between different dye concentrations (50-250 ppm) and percentage degradation of alizarin red (AR) dye by $0.025 \mathrm{~g}$ of nanomaterials $\mathrm{MH} 1$ and $\mathrm{MH} 5$ after $60 \mathrm{~min}$ of photocatalytic activity.

The decreased degradation at higher AR dye concentrations is ascribed to the adsorption of more dye molecules on the surface of the catalyst. This results in a decrease in the number of active sites on the catalyst surface. Additionally, an increase in dye concentration causes a decrease in the number of photons reaching the surface of catalysts, resulting in less UV light available for the exciton of catalyst particles due to more possible absorption by dye molecules.

\subsubsection{Effect of $\mathrm{pH}$ of the Medium}

The effect of $\mathrm{pH}$ of AR dye solution (200 ppm) on its percent degradation was investigated by changing the solution $\mathrm{pH}$ from acidic $(\mathrm{pH}=3,5)$, neutral $(7)$ and basic $(9,11)$ for photocatalytic activity with 60 min UV light irradiation (shown in Figure 10). The percentage degradations of different AR dye solutions with different $\mathrm{pH}$ were calculated for (MH1-MH5) nanomaterials and are given in Table 8. The percentages of dye degradation were found to be increased with increasing solution $\mathrm{pH}$ of AR from acidic to basic medium. Almost 25-29\% dye degradation was observed at acidic $\mathrm{pH}$ (3 and 5). The percentage degradation of AR was increased up to $48-50 \%$ with a neutral medium ( $\mathrm{pH} 7$ ) of dye solution. In total, $64-79 \%$ dye degradation was observed at basic $\mathrm{pH}(9,11)$ of dye solution. The highest dye degradation $(76.97 \%$ and $78.51 \%$ ) was achieved after $60 \mathrm{~min}$ irradiation of UV light for $\mathrm{pH} 11$ of dye solution by catalysts MH4 and MH5. The correlation between the percentage of dye degradation and $\mathrm{pH}$ of AR dye solution was found to be significant as the $\mathrm{R}^{2}$ value calculated was close to 1 (Figure 11). The correlation between the percentage of dye degradation and different $\mathrm{pH}$ of dye solution for MH1-MH3 are presented in Figure S8 (supplemental data). MH5 was an efficient catalyst, among all showing more than $80 \%$ dye degradation for all the factors studied, indicating the positive influence on degradation by increasing $\mathrm{Cr}$ doping. 
Table 8. Photocatalytic degradation of alizarin red (AR) dye (200 ppm) by MH1-MH5 (0.02 g) after $60 \mathrm{~min}$ of photocatalytic activity.

\begin{tabular}{cccccc}
\hline pH & MH 1 & MH 2 & MH 3 & MH 4 & MH 5 \\
\hline $\mathbf{3}$ & 25.62 & 26.20 & 26.86 & 25.92 & 26.14 \\
$\mathbf{5}$ & 27.69 & 27.11 & 28.29 & 28.68 & 27.88 \\
$\mathbf{7}$ & 49.22 & 48.01 & 49.78 & 49.89 & 50.22 \\
$\mathbf{9}$ & 64.63 & 64.98 & 65.22 & 65.18 & 67.63 \\
$\mathbf{1 1}$ & 76.71 & 76.02 & 76.10 & 76.97 & 78.51 \\
\hline
\end{tabular}

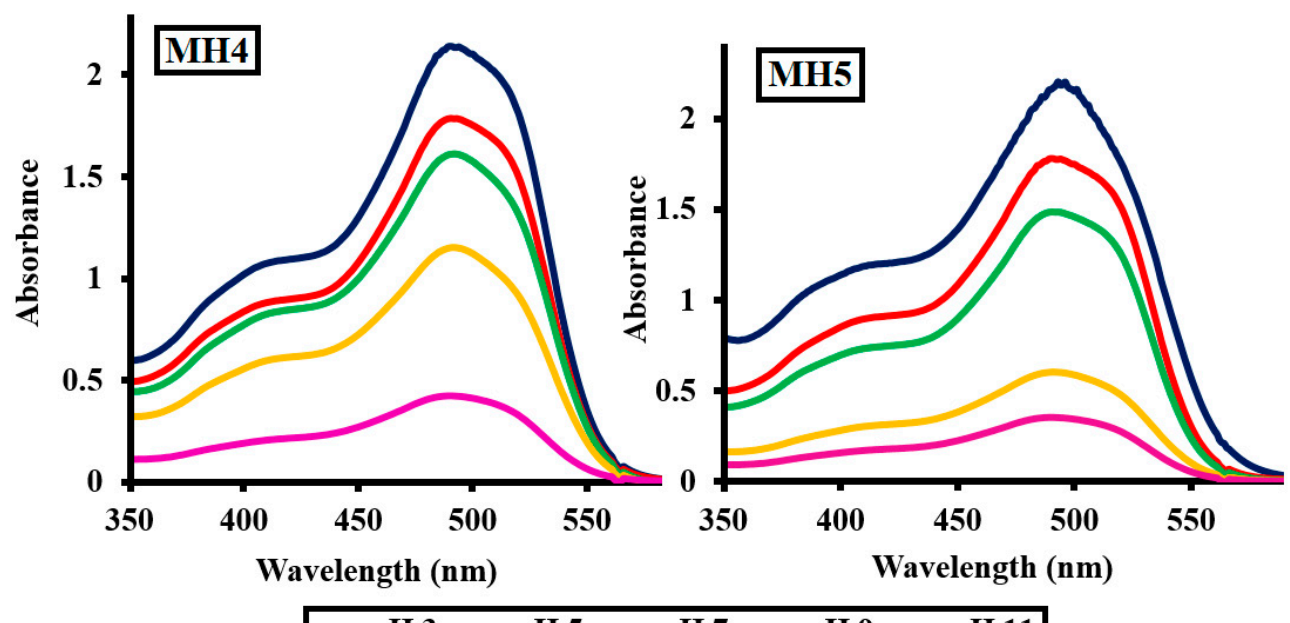

- pH $3-$ pH $5-$ pH $7-$ pH $9-$ pH 11

Figure 10. Photocatalytic degradation of alizarin red (AR) dye (250 ppm) after 60 min employing different $\mathrm{pH}$ by $0.025 \mathrm{~g}$ of $\mathrm{MH} 1$ and $\mathrm{MH} 5$.

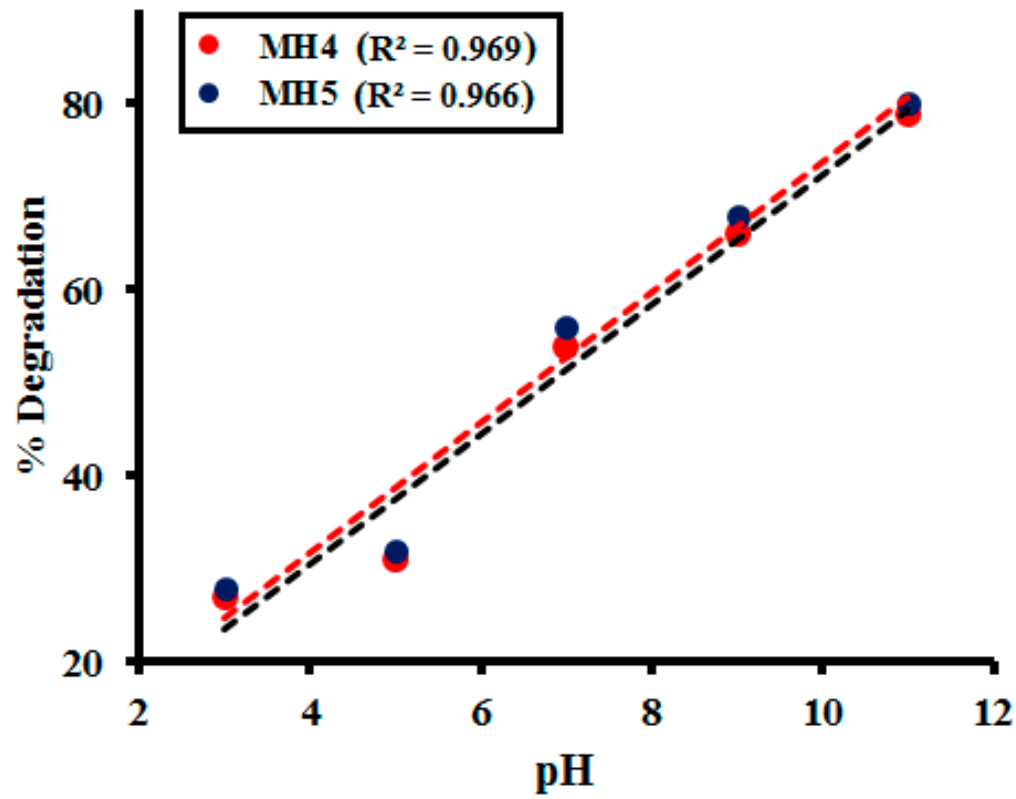

Figure 11. Correlation between different $\mathrm{pH}$ and percentage degradation of alizarin red (AR) dye (200 ppm) by $0.02 \mathrm{~g}$ of nanomaterials MH1 and MH5 after 60 min of photocatalytic activity.

\subsection{Kinetic Study}

Pseudo kinetic models, such as pseudo-first-order kinetics and pseudo-second-order, are frequently used to explain the adsorption kinetics of solid/liquid systems [36-38]. The kinetics of dye degradation 
on the surface of nanomaterials (MH1-MH5) was illustrated best by the pseudo-second-order kinetic model. The equation of the pseudo-second-order reaction is as follows:

$$
\mathrm{t} / \mathrm{C}_{\mathrm{t}}=1 / \mathrm{k}_{2} \mathrm{C}_{\mathrm{t}}^{2}+\mathrm{t} / \mathrm{C}_{\mathrm{o}}
$$

Pseudo-second order chemical reaction kinetics of dye degradation at different time intervals provides the best correlation of the experimental data, which are close to the unity depicted in Figure 12, and the reaction mechanism is found to be chemically rate-controlling [36]. As UV-light falls upon the metal oxide catalyst, electrons (e-) of the nanoparticles are excited from the valence band (VB) to the conduction band $(\mathrm{CB})$, which results in generating the positive hole $(\mathrm{h}+)$ in the valence band. The electrons in the $C B$ or stored in nanorods are trapped by the $\mathrm{O}_{2}$ molecule to form a reactive superoxide anion radical $\left(\mathrm{O}^{2-}\right)$. In contrast, the positive holes present in the VB react with water molecule and form hydroxyl radicals $(\mathrm{OH})$. These created radicals are highly reactive toward the photo degradation of AR dye [8]. A proposed mechanism of AR dye degradation is illustrated in Figure 13.

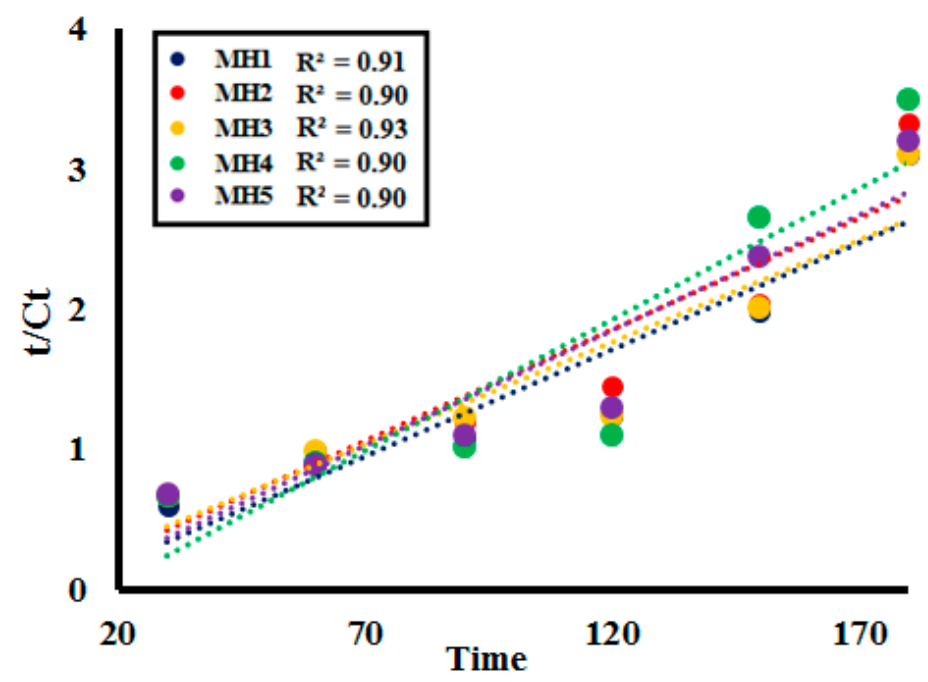

Figure 12. Pseudo second order kinetics of alizarin red (AR) dye degradation at different time intervals by nanomaterials (MH1-MH5).

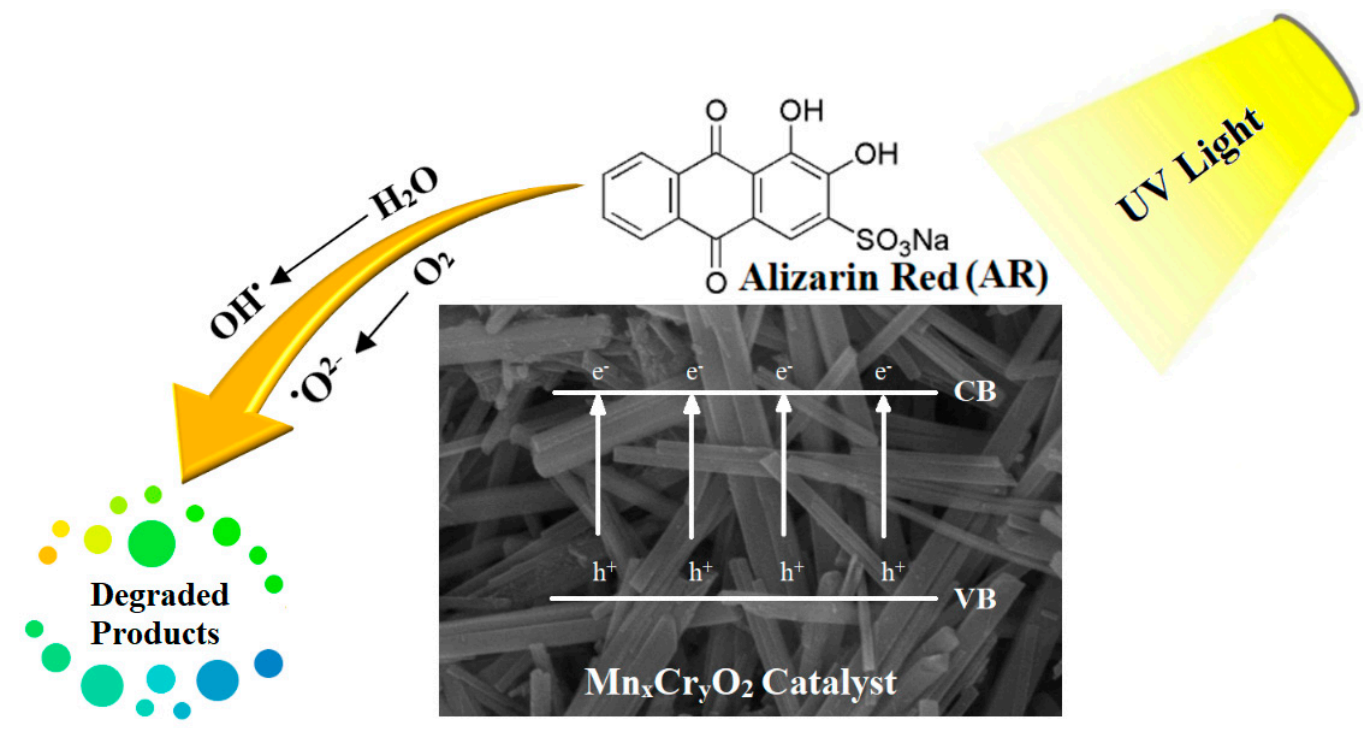

Figure 13. Proposed mechanism of AR dye degradation over surface of catalyst. 
For all the factors, the correlation was found for dye concentration after $60 \mathrm{~min}$ or photocatalytic activity under UV light with the factor studied enlisted in Table 9. Values of correlation constant $\mathrm{R}^{2}$ at different time intervals of photocatalytic activity were close to unity, as shown in Figure S9 of the supplementary data. Values of correlation constant $\mathrm{R}^{2}$ for different catalyst doses, dye concentrations, and $\mathrm{pH}$ values were close to unity, as shown in Figures S10-S12 (supplemental data).

Table 9. Correlation ( $\mathrm{R}^{2}$ Values) between factor studied and AR dye concentration after 60 min of photocatalytic activity ( $R^{2}$ Values).

\begin{tabular}{|c|c|c|c|c|}
\hline \multirow{2}{*}{ Samples } & \multicolumn{4}{|c|}{$\begin{array}{l}\text { Correlation between Factor Studied and AR Dye Concentration after } \\
60 \text { min of Photocatalytic Activity }\left(R^{2} \text { Values }\right)\end{array}$} \\
\hline & Time & Catalyst Dose & Dye Concentration & $\mathrm{pH}$ \\
\hline MH1 & 0.959 & 0.969 & 0.932 & 0.973 \\
\hline MH2 & 0.94 & 0.968 & 0.928 & 0.972 \\
\hline МH3 & 0.932 & 0.953 & 0.960 & 0.971 \\
\hline MH4 & 0.946 & 0.943 & 0.954 & 0.865 \\
\hline MH & 0.986 & 0.924 & 0.988 & 0.869 \\
\hline
\end{tabular}

\section{Materials and Methods}

\subsection{Chemicals and Reagents}

$\mathrm{KMnO}_{4}, \mathrm{~K}_{2} \mathrm{Cr}_{2} \mathrm{O}_{7}, \mathrm{HCl}$ and AR dye were purchased from Sigma Aldrich (Saint Louis, MO, USA). All the chemicals were of analytical grade and used without further purification.

\subsection{Synthesis of Chromium Manganese Oxide Nanomaterials (MH1-MH5)}

The MH1 sample was synthesized as $0.948 \mathrm{~g}$ of $\mathrm{KMnO}_{4}$ and $0.24 \mathrm{~g}$ of $\mathrm{K}_{2} \mathrm{Cr}_{2} \mathrm{O}_{7}$ were dissolved in $50 \mathrm{~mL}$ of water and shifted to a Teflon line parr reactor. In total, $20 \mathrm{~mL}$ of $\mathrm{HCl}$ was added subsequently to the mixture. The reaction mixture was stirred for $30 \mathrm{~min}$ and the parr reactor was sealed at heated at $180^{\circ} \mathrm{C}$ for $24 \mathrm{~h}$. After, the heat treatment reactor was cooled down to the room temperature and opened. A dark brown powdered solid was obtained at the base of reactor. The powdered solid was filtered off and washed with adequate water. The MH2-MH5 samples were prepared by the same method used for $\mathrm{MH} 1$ by using $0.41,0.35,0.18$ and $0.30 \mathrm{~g}$ of $\mathrm{K}_{2} \mathrm{Cr}_{2} \mathrm{O}_{7}$ for synthesis, respectively. The synthesized MH1-MH5 were obtained as a dark brown powder and were characterized and utilized for catalytic studies. Their found unit formula, powder X-ray diffraction (PXRD) peaks and composition (by energy dispersive $\mathrm{X}$-ray (EDX)), along with the synthetic conditions, are summarized in Table 1.

\subsection{Characterization Techniques}

Powder X-ray diffraction (PXRD) measurements were performed in an X-ray diffractometer (Bruker, AXS D8; Yokohama-shi, Japan) with Cu-K $\alpha$ radiation (1.5406 $\AA$ ) at a scanning rate of $10^{\circ}$ per min in the $2 \theta$ range from $10^{\circ}$ to $90^{\circ}$. The morphological features of catalysts were characterized by a scanning electron microscope (SEM) (JEOL, JSM-6360 EO; Tokyo, Japan), and the elemental composition was estimated by an energy dispersive X-ray (EDX) diffractometer (JEOL JSM-6360 LV; Tokyo, Japan). The surface area and pore structure parameters were calculated by a Brunauer-Emmett-Teller (BET) analysis performed through Quantachrome Nova 2200e (Tokyo, Japan). A sonicator (Bransonic 2510EMT bath; Darmstadt, Germany) was used for the surface area deployment of nanomaterials. A PTFE(polytetrafluoroethylene)-lined, Stainless Steel, Parr 5521 high-pressure compact Autoclave was used for the temperature and pressure treatments. A UV lamp (length: $288 \mathrm{~mm}$, pipe diameter: $16 \mathrm{~mm}$, voltage: $220 \mathrm{~V}$, power; $8 \mathrm{~W}$, wavelength range: $240-285 \mathrm{~nm}$ ) was used as a light source for photocatalytic alizarin red (AR) dye degradation activity. The catalytic dye degradation was studied by an ultraviolet (UV) spectrophotometer (Shimadzu; Osaka, Japan) in the frequency range of 250-800 nm. 


\subsection{Photocatalytic Activity}

Photocatalytic alizarin red (AR) dye degradation was conducted according to previous reports $[19,39,40]$ with some modifications. A 200 ppm solution of AR dye was prepared in water. Blank experiments were conducted for the AR dye solution without the presence of catalyst. One of the blank experiments was conducted by keeping the sample of dye solution for $180 \mathrm{~min}$ without UV light irradiation and the absorption spectra were taken at different time intervals. Another experiment was done by irradiating the dye solution for $180 \mathrm{~min}$ and the UV spectra were measured at various time intervals by taking sample aliquots.

To measure the photocatalytic activity in the presence of a catalyst, $50 \mathrm{~mL}$ of $200 \mathrm{ppm}$ AR dye solution was subjected to stirring in beaker under UV light source in the presence of $0.02 \mathrm{~g}$ catalysts (MH1). Aliquots of $5 \mathrm{~mL}$ from reaction mixture were taken at different time intervals of $0,30,60,90,120$, 150 and $180 \mathrm{~min}$. is Catalysts in sample aliquots were spun down by a centrifuge for $1 \mathrm{~min}$ and were separated from reaction mixture. The separated catalyst was not added back to the reaction mixture. After the separation of the catalyst, the UV spectrum was measured from the UV spectrophotometer for all the taken aliquots at different time intervals. From the spectrum, absorbance at $501 \mathrm{~nm}$ was noted and the percentage degradation of AR was calculated for all the sample aliquots by following Equation (5) [39].

$$
\% \text { Degradation }=\mathrm{C}_{\mathrm{o}}-\mathrm{C}_{\mathrm{t}} / \mathrm{C}_{\mathrm{o}} \times 100
$$

where $C_{o}$ is the initial concentration and $C_{t}$ is the concentration at different sampling times. The concentration of AR after the reaction time was measured by the calibration curve between the absorbance and known AR concentration. The unknown AR concentration for the measured absorbance at different time intervals was calculated by the linear equation of known data. The same method was adopted to check the catalytic activity for all other catalysts (MH2-MH5).

The percentage degradation of the AR dye was calculated by variating the initial dye concentration $(50,100,150,200$ and $250 \mathrm{ppm})$, the change in catalyst dose $(0.015,0.02,0.025,0.03 \mathrm{~g}, 0.035 \mathrm{~g} 0.040 \mathrm{~g})$ and the $\mathrm{pH}$ of the dye solution $(3,5,7,9,11)$ after $60 \mathrm{~min}$ of photocatalytic activity under UV light irradiation by following the same procedure as described above. To study the effect of different $\mathrm{pHs}$ of the AR dye solution, the $\mathrm{pH}$ was adjusted by employing $\mathrm{HCl}$ for an acidic $\mathrm{pH}$ and $\mathrm{NaOH}$ for a basic medium.

\section{Conclusions}

The presented work describes photocatalytic AR dye degradation by a series of Cr-based manganese oxide nanomaterials (MH1-MH5). All the catalysts have shown more than $70 \%$ AR dye degradation, exhibiting the good catalytic efficiencies for dye removal. MH1 and MH5 showed more than $85 \%$ dye degradation for the effect of time intervals, whereas MH4 and MH5 showed more than $80 \%$ dye degradation for different catalyst doses. MH3 and MH5 showed more than $60 \%$ dye degradation for different dye concentrations employed. MH4 and MH5 showed more than 70\% dye degradation for different $\mathrm{pH}$ values of dye solution. The catalytic pathways were analyzed by applying the kinetic model. The pseudo second order was found to be the best fitted kinetic model, indicating a chemically rate controlled mechanism. Values of constant $R^{2}$ for all the factors studied were close to unity, depicting good correlation between the factors studied. MH5 was an efficient catalyst among all, showing more than $80 \%$ dye degradation for all the factors studied.

Supplementary Materials: The following are available online at http://www.mdpi.com/2073-4344/10/10/1150/s1. Figure S1: Energy dispersive X-ray (EDX) Spectra of Chromium Doped Manganese Oxide Nanomaterials (MH1-MH5), Figure S2: SEM Images of MH1, MH2, MH3, MH4, Figure S3(a): UV Absorption Spectrum of AR Dye; Figure S3(b): UV Absorption Spectra of AR Dye; Figure S4: Percent AR Dye Degradation at Different Time Intervals (30, 60, 90, 120, 150 and $180 \mathrm{~min}$ ) by Photocatalysts (MH1-MH5); Figure S5: Correlation between Time and \%Degradation of Alizarin Red (AR) Dye (200ppm) by 0.02g of Nanomaterials MH2-MH4; Figure S6: Correlation between \%Degradation of Alizarin Red (AR) Dye (200 ppm) and Different Catalyst Doses of MH1-MH3after 60min of Photocatalytic Activity; Figure S7: Correlation between \%Degradation of Alizarin Red (AR) Dye by 
0.02g of Nanomaterials MH1-MH3 at Different Dye Concentrations (50-250ppm); Figure S8: Correlation between $\%$ Degradation of Alizarin Red (AR) Dye (200ppm) by 0.02g of Nanomaterials MH1-MH3 and Different Time Intervals; Figure S9: Correlation between Alizarin Red (AR) Dye Concentration of Photocatalytic Activity by $0.02 \mathrm{~g}$ of Nanomaterials (MH1-MH5) at Different Time Intervals; Figure S10: Correlation between Alizarin Red (AR) Dye Concentration after 60min of Photocatalytic Activity by Nanomaterials (MH1-MH5) at Different Catalyst Doses; Figure S11: Correlation between Alizarin Red (AR) Dye Concentration after 60min of Photocatalytic Activity by Nanomaterials (MH1-MH5) at Different Dye Concentrations; Figure S12: Correlation between Alizarin Red (AR) Dye Concentration after 60min of Photocatalytic Activity by Nanomaterials (MH1-MH5) at Different $\mathrm{pH}$.

Author Contributions: Conceptualization, M.H. and A.A.A.; Methodology, M.H., S.K. and A.A.A.; Investigation, M.H., S.K., R.G. and N.R.; Resources, S.M., M.Z., S.A.A.S., Z.A.Z. and A.B.; Writing-Original Draft Preparation, M.H., A.A.A. and S.K. Writing-Review and Editing, A.A.A., S.M., N.R. and R.G.; Funding Acquisition, A.A.A. and Z.A.Z. Project Administration, A.A.A. and Z.A.Z. All authors have read and agreed to the published version of the manuscript.

Funding: This research was mainly financially supported by Higher Education Commission Islamabad, Pakistan under HEC-NRPU project No. 6492.

Acknowledgments: The authors would also like to acknowledge the Ministry of Higher Education (MOHE) Malaysia for financial support under the Fundamental Research Grant Scheme (FRGS) with sponsorship reference numbers FRGS/1/2019/STG05/UITM/02/9. The author would also like to acknowledge Universiti Teknologi MARA for the financial support under the reference number 600-IRMI/FRGS 5/3 (424/2019).

Conflicts of Interest: Authors have no conflict of interest.

\section{References}

1. Jain, R.; Sharma, S.; Manoj, S.; Bansal, S.; Gupta, K. Application of titanium dioxide semiconductor photocatalysis in the photomineralization of dyes in textile industry effluents. Indian J. Environ. Prot. 2004, 23, 63-68.

2. Natarajan, S.; Bajaj, H.C.; Tayade, R.J. Recent advances based on the synergetic effect of adsorption for removal of dyes from waste water using photocatalytic process. J. Environ. Sci. 2018, 65, 201-222. [CrossRef] [PubMed]

3. Oller, I.; Malato, S.; Pérez, J.S. Combination of Advanced Oxidation Processes and biological treatments for wastewater decontamination-A review. Sci. Total Environ. 2011, 409, 4141-4166. [CrossRef] [PubMed]

4. Li, Z.; Liu, J.; Zhang, F.-J.; Oh, W.-C. UV and visible light photodegradation effect on Fe-CNT/TiO 2 composite catalysts. Bull. Mater. Sci. 2013, 36, 293-299. [CrossRef]

5. Hussain, K.I.; Usman, M.; Siddiq, M.; Rasool, N.; Nazar, M.F.; Ahmad, I.; Holder, A.A.; Altaf, A.A. Application of micellar enhanced ultrafiltration for the removal of sunset yellow dye from aqueous media. J. Dispersion Sci. Tech. 2017, 38, 139-144. [CrossRef]

6. Ponnusamy, V.; Lakshmi, G. Enhanced photocatalytic performance of $\left(\mathrm{ZnO} / \mathrm{CeO}_{2}\right)-\beta-\mathrm{CD}$ system for the effective decolorization of Rhodamine B under UV light irradiation. Appl. Water Sci. 2017, 7, 4025-4036. [CrossRef]

7. Mantzavinos, D.; Psillakis, E. Enhancement of biodegradability of industrial wastewaters by chemical oxidation pre-treatment. J. Chem. Technol. Biotechnol. 2004, 79, 431-454. [CrossRef]

8. Saeed, K.; Zada, N.; Khan, I. Photocatalytic degradation of alizarin red dye in aqueous medium using carbon nanotubes/Cu-Ti oxide composites. Sep. Sci. Technol. 2018, 54, 2729-2737. [CrossRef]

9. Saroj, S.; Kumar, K.; Pareek, N.; Prasad, R.; Singh, R. Biodegradation of azo dyes Acid Red 183, Direct Blue 15 and Direct Red 75 by the isolate Penicillium oxalicum SAR-3. Chemosphere 2014, 107, 240-248. [CrossRef]

10. Iqbal, M.J.; Ashiq, M.N. Thermodynamics of adsorption of dyes from aqueous media on activated charcoal. J. Res. Sci. 2007, 18, 91-99.

11. Rauf, M.; Ashraf, S. Radiation induced degradation of dyes-An overview. J. Hazard. Mater. 2009, 166, 6-16. [CrossRef]

12. Akpan, U.; Hameed, B. Parameters affecting the photocatalytic degradation of dyes using $\mathrm{TiO}_{2}$-based photocatalysts: A review. J. Hazard. Mater. 2009, 170, 520-529. [CrossRef] [PubMed]

13. Qu, X.; Alvarez, P.J.J.; Li, Q. Applications of nanotechnology in water and wastewater treatment. Water Res. 2013, 47, 3931-3946. [CrossRef] [PubMed]

14. Nakata, K.; Fujishima, A. $\mathrm{TiO}_{2}$ photocatalysis: Design and applications. J. Photochem. Photobiol. C Photochem. Rev. 2012, 13, 169-189. [CrossRef] 
15. Arias, N.P.; Dávila, M.T.; Giraldo, O.H. Electrical behavior of an octahedral layered OL-1-type manganese oxide material. Ionics 2012, 19, 201-214. [CrossRef]

16. Giraldo, O.; Arias, N.; Becerra, M. Electrical properties of $\mathrm{TiO}_{2}$-pillared bidimensional manganese oxides. Appl. Clay Sci. 2017, 141, 157-170. [CrossRef]

17. Niederberger, M.; Pinna, N. Metal Oxide Nanoparticles in Organic Solvents; Springer Science and Business Media: New York, NY, USA, 2009.

18. Arami, M.; Limaee, N.Y.; Mahmoodi, H.; Tabrizi, N.S. Removal of dyes from colored textile wastewater by orange peel adsorbent: Equilibrium and kinetic studies. J. Colloid Interface Sci. 2005, 288, 371-376. [CrossRef]

19. Kausar, S.; Altaf, A.A.; Hamayun, M.; Rasool, N.; Hadait, M.; Akhtar, A.; Muhammad, S.; Badshah, A.; Shah, S.A.A.; Zakaria, Z.A. i-Propylammonium Lead Chloride Based Perovskite Photocatalysts for Depolymerization of Lignin Under UV Light. Molecules 2020, 25, 3520. [CrossRef]

20. Danish, M.; Tayyab, M.; Akhtar, A.; Altaf, A.A.; Kausar, S.; Ullah, S.; Iqbal, M. Effect of soft template variation on the synthesis, physical, and electrochemical properties of Mn3O4 nanomaterial. Inorg. Nano-Metal Chem. 2020, 1-7. [CrossRef]

21. Altaf, A.A.; Ahmed, M.; Hamayun, M.; Kausar, S.; Waqar, M.; Badshah, A. Titania nano-fibers: A review on synthesis and utilities. Inorg. Chim. Acta 2020, 501, 119268. [CrossRef]

22. Duan, J.; Zheng, Y.; Chen, S.; Tang, Y.; Jaroniec, M.; Qiao, S.Z. Mesoporous hybrid material composed of $\mathrm{Mn3 \textrm {O } 4 n a n o p a r t i c l e s ~ o n ~ n i t r o g e n - d o p e d ~ g r a p h e n e ~ f o r ~ h i g h l y ~ e f f i c i e n t ~ o x y g e n ~ r e d u c t i o n ~ r e a c t i o n . ~}$ Chem. Commun. 2013, 49, 7705-7707. [CrossRef] [PubMed]

23. Sood, S.; Mehta, S.K.; Umar, A.; Kansal, S.K. The visible light-driven photocatalytic degradation of Alizarin red $\mathrm{S}$ using Bi-doped $\mathrm{TiO}_{2}$ nanoparticles. New J. Chem. 2014, 38, 3127-3136. [CrossRef]

24. Sun, J.; Lu, H.; Du, L.; Lin, H.; Li, H. Anodic oxidation of anthraquinone dye Alizarin Red S at Ti/BDD electrodes. Appl. Surf. Sci. 2011, 257, 6667-6671. [CrossRef]

25. Joshi, K.; Shrivastava, V. Degradation of alizarine red-S (A textiles dye) by photocatalysis using ZnO and $\mathrm{TiO}_{2}$ as photocatalyst. Int. J. Environ. Sci. 2011, 2, 8-21.

26. Kansal, S.K.; Lamba, R.; Mehta, S.; Umar, A. Photocatalytic degradation of Alizarin Red S using simply synthesized ZnO nanoparticles. Mater. Lett. 2013, 106, 385-389. [CrossRef]

27. Jagodzinski, H. The Role of Munich in X-Ray Crystallography and the Development of Powder Diffraction. Mater. Sci. Forum 1991, 79, 1-16. [CrossRef]

28. Cullity, B.; Stock, S. Elements of X-ray Diffraction, 3rd ed.; Prentice Hall: New York, NY, USA, 2001; pp. $174-177$.

29. Aé, L.; Kieven, D.; Chen, J.; Klenk, R.; Rissom, T.; Tang, Y.; Lux-Steiner, M.C.; Rissom, T. ZnO nanorod arrays as an antireflective coating for $\mathrm{Cu}(\mathrm{In}, \mathrm{Ga}) \mathrm{Se}_{2}$ thin film solar cells. Prog. Photovolt. Res. Appl. 2010, 18, $209-213$. [CrossRef]

30. Saleem, M. Effect of zinc acetate concentration on the structural and optical properties of $\mathrm{ZnO}$ thin films deposited by Sol-Gel method. Int. J. Phys. Sci. 2012, 7, 2971-2979. [CrossRef]

31. Bindu, P.; Thomas, S. Estimation of lattice strain in $\mathrm{ZnO}$ nanoparticles: X-ray peak profile analysis. J. Theor. Appl. Phys. 2014, 8, 123-134. [CrossRef]

32. Wang, B.; Iqbal, J.; Shan, X.; Huang, G.; Fu, H.; Yu, R.; Yu, D. Effects of Cr-doping on the photoluminescence and ferromagnetism at room temperature in $\mathrm{ZnO}$ nanomaterials prepared by soft chemistry route. Mater. Chem. Phys. 2009, 113, 103-106. [CrossRef]

33. Kernazhitsky, L.; Shymanovska, V.; Gavrilko, T.; Naumov, V.; Fedorenko, L.; Kshnyakin, V.; Burtsev, A.; Baran, J. Effect of Cr-Doping on Luminescence of Nanocrystalline Anatase $\mathrm{TiO}_{2}$ Powders. Ukr. J. Phys. 2016, 61, 482-488. [CrossRef]

34. Chu, J.; Sher, A. Device Physics of Narrow Gap Semiconductors; Springer Science and Business Media: New York, NY, USA, 2010.

35. Xu, F.; Yuan, Z.-Y.; Du, G.-H.; Ren, T.-Z.; Volcke, C.; Thiry, P.; Su, B.-L. A low-temperature aqueous solution route to large-scale growth of $\mathrm{ZnO}$ nanowire arrays. J. Non-Cryst. Solids 2006, 352, 2569-2574. [CrossRef]

36. Ho, Y.; McKay, G. Pseudo-second order model for sorption processes. Process Biochem. 1999, 34, 451-465. [CrossRef]

37. Ho, Y.; McKay, G. Kinetic Models for the Sorption of Dye from Aqueous Solution by Wood. Process Saf. Environ. Prot. 1998, 76, 183-191. [CrossRef]

38. Liu, Z.; Zhang, F.-S. Removal of lead from water using biochars prepared from hydrothermal liquefaction of biomass. J. Hazard. Mater. 2009, 167, 933-939. [CrossRef] 
39. Kansal, S.; Singh, M.; Sud, D. Studies on $\mathrm{TiO}_{2} / \mathrm{ZnO}$ photocatalysed degradation of lignin. J. Hazard. Mater. 2008, 153, 412-417. [CrossRef]

40. Kausar, S.; Altaf, A.A.; Hamayun, M.; Danish, M.; Zubair, M.; Naz, S.; Muhammad, S.; Zaheer, M.; Ullah, S.; Badshah, A. Soft template-based bismuth doped zinc oxide nanocomposites for photocatalytic depolymerization of lignin. Inorg. Chim. Acta 2020, 502, 119390. [CrossRef]

(C) 2020 by the authors. Licensee MDPI, Basel, Switzerland. This article is an open access article distributed under the terms and conditions of the Creative Commons Attribution (CC BY) license (http://creativecommons.org/licenses/by/4.0/). 